\title{
Trade-off Assessment on Two Steel Ball Brands Use at the Ball Mill Plant of a Ghanaian Mine
}

\author{
James Obiri-Yeboah ${ }^{1}$, Alex Kwaku Peprah ${ }^{2}$, Hope Nador ${ }^{3}$ \\ ${ }^{1}$ Unit Manager - Technical, Metallurgy Department, Goldfields Ghana Limited-Tarkwa Mine, Ghana \\ ${ }^{2}$ Lecture, Department of Computer Science, Catholic University of Ghana, Sunyani, Ghana \\ ${ }^{3}$ Processing Supervisor, Metallurgy Department, Goldfields Ghana Limited-Tarkwa Mine, Ghana \\ Correspondence: James Obiri-Yeboah, Unit Manager - Technical, Metallurgy Department, Goldfields Ghana \\ Limited-Tarkwa Mine, Ghana.
}

Received: June 13, 2017

doi:10.5539/ibr.v10n10p198

\author{
Accepted: September 7, $2017 \quad$ Online Published: September 25, 2017 \\ URL: https://doi.org/10.5539/ibr.v10n10p198
}

\begin{abstract}
Realistically, this research shows that, the type or brand of input reagent such as steel ball is a vital parameter to be considered to ensure cost saving in mineral processing business. Logically, the study pointed out the shortfall in the acceptance of input reagent (steel ball) of a production system on only the unit price variance for different items. Clearly, the paper aims at closing the lack of information gap existing in the Ghanaian mining company to overcome the situation of compromising efficiency of the plant production whilst maximizing profit. Furthermore, assessing the overall effect by taking into consideration the operating variables, painted a pragmatic and reliable picture of the prevailing scenario. Consequently, company 1 with a mean discharge product of $49.42 \%$ passing $150 \mu \mathrm{m}$ was at a cost of US $\$ 1.68$ whiles company 2 with mean discharge product of $50.12 \%$ passing $150 \mu \mathrm{m}$ was at a cost of US\$1.30. Comparatively, company 2 brand of steel ball usage gave an overall trade-off of $0.8 \%$ as against the usage of company 1 steel ball brand. The paper recommended the use of company 2 steel ball brand as a cost saving enhancement decision for gold production in the Ghanaian Mine.
\end{abstract}

Keywords: percentage passing, milling, retain, micron, steel ball brand

\section{Introduction}

Trade-off is the gain in accepting less or sacrifice one commodity or item for the other. Cautious and logical evaluation of trade-off involves comparing cost and benefit of available alternatives with each other. Economically, trade-off expresses the opportunity cost in most favorite potential alternative (Johnson, 2005; Hill, 2015). This includes, undertaking cost saving assessment that focus on optimizing cost in an endeavour. Cost saving initiative is the decision or action that will result in the fulfillment of a lower cost objective than the historical cost or the projected cost of an item (Hornby, 2010). Invariably, to enhance cost saving initiative, element-by-element examination of the estimated or actual cost of contract performance or item being used cannot be overemphasized. The goal is to form an opinion on whether the proposed costs are in line with pragmatic and efficient performance cost (Weisbrod and Hansen, 1981). At the Carbon in leach (C.I.L) Plant of the Ghanaian mine in this research, there are several cost centers with cost elements of distinctive total cost dependency. Steel ball consumption implies the amount of steel ball used in a particular time period to enhance grinding of ore in tumbling Mill (a designed drum which use rotational motion to generate abrasive and impact forces between steel balls and ore to effect ore grinding). At the end of grinding process, the steels are completely destroyed whiles the ore break down into relatively finer particle size fractions as compared to the ore size (feed ore size) before the start of grinding process. Obviously, the amount of steel ball use in achieving a given size particle is a function of the level at which a give steel ball type can withstand the destruction effect of a particular ore. This trade-off assessment on steel ball consumption seeks to establish the cost effectiveness of the steel ball brand for the milling operation at the C.I.L Plant. Additionally, the high cost of steel balls has a direct effect on the increasing cost of production at the C.I.L plant process. This paper is to close the lack of information (i.e. information on cost effective steel ball brand) gap existing in the Ghana mine. This will help the company to overcome the situation of compromising efficiency of the plant by trying to maximize profit under pretext of cheap price. The framework of this research covers, quantitative evaluation of the use of two different brands of steel balls in sequential order as input reagent at plant condition for 30 days period. The paper aims at selecting a 
cost effective steel ball brand for the mineral process plant of the Ghanaian mine in this research. Applicably, Bertsimas, Farias and Trichakis (2012), assesses the problem of designing the right objective which resolves the normal difficulty of selecting between efficiency and fairness in the perspective of a framework that covers a number of resource allocation under the topic On the Efficiency-Fairness Trade-off.

\subsection{Cost Factors and Analysis at Steel Ball Consumption at the Ghanaian Mine Processing Plant}

The processing plant of the Ghanaian mine being under studied, operates at a throughput capacity of 1,532 ton of ore per hour with an expected daily production of 36,000 tones at 23.5 hours plant availability. The input elements (reagents) for ore grinding process at the Ball Mill plant are electric power and two brands $(60 \mathrm{~mm}$ steel ball of company 1 and 2). The cost of Ball Mill power usage is given as US\$ 0.17 for kilowatt hour per ton of ore milled whiles the company 1 and 2 steel ball brands cost US $\$ 1237$ and US $\$ 1338$ per ton of steel balls respectively (Technical Report, 2016). Critically, it will be suicidal to select any alternative of reagent without given thorough assessment to it effect on the total production cost of plant operation. Therefore, the seemingly lower unit cost and a supplier's quality indication of one reagent as compared to another, will not necessarily justify its selection for usage. Imperatively, cost allocation, cost-effectiveness analysis, and cost-benefit analysis are ranges of cost analysis methods for evaluating steel ball consumption efficiency at the processing plant operation. They range from fairly simple program-level methods to highly technical and specialized methods. Cost allocation is a simpler concept than either cost-benefit analysis or cost-effectiveness analysis. At the processing plant operation, it means setting up budgeting and accounting systems in a way that allows managers to determine a unit cost of service gain out of a given type of steel ball. This information is mostly a management tool that focuses on decision making. However, since the units measured are also outcomes of interest to evaluators, cost allocation provides some of the basic information needed to conduct more determined cost analyses (Kettner, Moroney \& Martin, 1990; Zeckhauser, 1975). In furtherance of authenticating this assessment, power consumption and end product particle size of the different brands of steel ball used were evaluated to point out the tradeoff that may be obtained in using a particular type of steel ball. This gives a holistic assessment that has the potential of given a reliable selection of the brand of steel ball require for efficient plant operation. Hence, at the processing plant operation, the amount of steel balls use to transform a given quantity of ore to a finer grind size fraction, measures the steel ball consumption rate. The consumption rate is then achieved by relating a given mass quantity of steel balls use to grind a unit mass quantity of ore. The unit expression of steel ball consumption rate is given as gram per ton of ore $(\mathrm{g} / \mathrm{t})$ or kilogram per ton of ore $(\mathrm{Kg} / \mathrm{t})$. Furthermore, power usage in terms of kilowatt hour per ton of ore grinded and percentage particle size passing through a 150 micron $(\mu \mathrm{m})$ aperture screen target, are the parameters for evaluating performance of a given steel ball brand used in grinding process.

\section{Material and Method Used}

Fundamentally, the insufficiency of information on the trade-off of steel ball usage at the process plant is a recipe for production deficiencies. Therefore, monthly production report records of the two different brands (i.e. company 1 and 2) of steel balls, used under constant plant operating conditions were studied to ascertain their respective opportunity cost with regards to end product as a target. According to Young (2013), comparison matrix is a useful tool for establishing criteria priorities of the given opportunities. Basically, from Young's point of view, these criteria priorities are formed out of weighted scores generated from recorded values of the items under consideration. This paper points to undertaking holistic evaluation of all the related parameters to arrive at practical trade-off for the two steel ball brands. The steel balls under consideration in this research, have equal parameters that relate to the wear rate. Hence cost analysis technic of unit cost evaluation was used to ascertain indices of the two steel ball brands for the process plant. Additionally, the power draw trends from a Scada system (i.e. automatic control and recording system) were used to evaluate the power consumption levels of the different steel ball brands. Saunder, lewis and Thornhill, (2009), affirmed the examination of simple index numbers as suitable method for comparing relative changes of different unit variables for drawing pragmatic conclusion. Hence, from the production reports, different brands of steel balls and their corresponding discharge product variables, call for the use of quantitative analysis of simple index numbers as the appropriate model for trade-off evaluations. Therefore, quantitative evaluations of power usage, steel ball consumption rate and corresponding effects of the different steel ball brands on the particle size fractions produced were obtained to enhance realistic conclusions.

\section{Result from Daily Operational Report Data}

Operational reports for 30 days usage of $60 \mathrm{~mm}$ steel balls of each brand (Steel Ball Company 1 and 2) in the Ball Mill at controllable amount at mill power set point of not less than $11000 \mathrm{KWh}$ were studied. The monthly 
production report parameters use for this assessment includes the daily records of steel ball consumption, mill power usage and tonnage milled with corresponding throughput records. Additionally, Laboratory tested particle size fractions for percentage retained on $150 \mu \mathrm{m}$ aperture screen $(+150 \mu \mathrm{m}$ particle size fraction) for Ball Mill feed and discharge were monitored throughout the 30 days period (Technical Report, 2016). These parameters were subjected to appropriate quantitative analysis to generate the needed indications towards the trade-off focus. The tables 1 and 2 were the results obtained from the daily report of the said periods for the two brands of steel balls. The tables show appropriately the summation of daily steel ball consumption and milled tonnage per day with average values for power draw, throughput, Ball Mill feed and discharge in line with pragmatic decision considerations.

Table 1. Company 1 Steel ball daily plant operating report

\begin{tabular}{|c|c|c|c|c|c|c|c|}
\hline No. Day & Daily Steel Ball & Power & Daily & Throughput, & Ball Mill & Feed & Average $\quad$ Ball \\
\hline & $\begin{array}{l}\text { Consumption } \\
(\mathrm{Kg})\end{array}$ & $\begin{array}{l}\text { Draw, } \\
\text { KWh }\end{array}$ & $\begin{array}{l}\text { milled } \\
\text { tonnes, } \mathrm{t}\end{array}$ & & $+150 \mu \mathrm{m}$ & & $\begin{array}{l}\text { Mill Discharge } \\
+150 \mu \mathrm{m}, \%\end{array}$ \\
\hline 1 & 30,000 & 11,983 & 24,929 & 1275 & 75.52 & & 62.27 \\
\hline 2 & 50,000 & 11,841 & 36,102 & 1504 & 74.49 & & 58.64 \\
\hline 3 & 40,000 & 11,909 & 37,192 & 1550 & 81.05 & & 68.85 \\
\hline 4 & 40,000 & 11,965 & 35,321 & 1472 & 77.46 & & 61.57 \\
\hline 5 & 55,000 & 11,966 & 33,944 & 1414 & 79.55 & & 54.43 \\
\hline 6 & 35,000 & 11,932 & 32,772 & 1366 & 81.63 & & 59.87 \\
\hline 7 & 40,000 & 11,899 & 35,299 & 1471 & 84.23 & & 61.76 \\
\hline 8 & 40,000 & 12,023 & 33,901 & 1414 & 80.71 & & 50.78 \\
\hline 9 & 40,000 & 11,975 & 36,255 & 1511 & 82.42 & & 60.49 \\
\hline 10 & 30,000 & 11,962 & 34,735 & 1447 & 78.95 & & 58.71 \\
\hline 11 & 30,000 & 11,895 & 34,727 & 1447 & 69.88 & & 55.59 \\
\hline 12 & 30,000 & 11,828 & 34,719 & 1521 & 78.07 & & 59.44 \\
\hline 13 & 15,000 & 11,748 & 14,175 & 1284 & 77.93 & & 65.31 \\
\hline 14 & 6,000 & 11,544 & 32,374 & 1494 & 78.95 & & 62.26 \\
\hline 15 & 50,000 & 11,377 & 32,937 & 1386 & 80.34 & & 50.58 \\
\hline 16 & 40,000 & 11,395 & 37,266 & 1553 & 77.32 & & 56.13 \\
\hline 17 & 60,000 & 11,626 & 35,575 & 1592 & 80.34 & & 55.11 \\
\hline 18 & 47,000 & 11,873 & 36,708 & 1530 & 82.92 & & 61.32 \\
\hline 19 & 43,000 & 11,980 & 37,218 & 1559 & 81.79 & & 61.67 \\
\hline 20 & 35,000 & 11,975 & 36,364 & 1515 & 81.47 & & 57.21 \\
\hline 21 & 28,000 & 11,925 & 33,459 & 1404 & 81.18 & & 67.26 \\
\hline 22 & 33,000 & 11,739 & 38,595 & 1608 & 80.36 & & 65.06 \\
\hline 23 & 34,000 & 11,783 & 36,020 & 1580 & 77.63 & & 68.58 \\
\hline 24 & 36,000 & 11,725 & 30,509 & 1278 & 78.21 & & 53.03 \\
\hline 25 & 28,000 & 11,959 & 36,617 & 1526 & 79.11 & & 55.80 \\
\hline 26 & 26,000 & 11,772 & 35,686 & 1564 & 63.03 & & 59.53 \\
\hline 27 & 52,000 & 11,929 & 31,653 & 1319 & 81.16 & & 60.55 \\
\hline 28 & 25,000 & 11,775 & 29,693 & 1242 & 78.48 & & 57.80 \\
\hline 29 & 45,000 & 11,675 & 31,759 & 1362 & 82.19 & & 56.10 \\
\hline 30 & 30,000 & 11,875 & 28,415 & 1193 & 83.83 & & 53.03 \\
\hline AVERAGE/SUM & 1093000 & 11828 & 1004920 & 1446 & 79.23 & & 59.29 \\
\hline
\end{tabular}


Table 2. Company 2 Steel Ball Daily Operation Report

\begin{tabular}{|c|c|c|c|c|c|c|}
\hline No. Day & $\begin{array}{l}\text { Daily Steel } \\
\text { Ball } \\
\text { Consumption } \\
(\mathrm{Kg})\end{array}$ & $\begin{array}{l}\text { Power } \\
\text { Draw, } \\
\text { KWh }\end{array}$ & $\begin{array}{l}\text { Daily } \\
\text { milled } \\
\text { tonnes, } \\
\text { t }\end{array}$ & Throughput, t/h & $\begin{array}{l}\text { Ball Mill Feed } \\
+150 \mu \mathrm{m}, \%\end{array}$ & 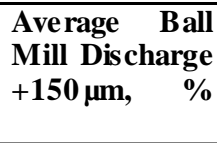 \\
\hline 1 & 20,000 & 11,498 & 34,248 & 1427 & 81.51 & 70.67 \\
\hline 2 & 20,000 & 11,469 & 34,211 & 1425 & 81.21 & 66.38 \\
\hline 3 & 20,000 & 11,406 & 35,667 & 1486 & 78.01 & 64.46 \\
\hline 4 & 15,000 & 11,464 & 28,823 & 1241 & 81.96 & 65.11 \\
\hline 5 & 20,000 & 11,462 & 31,400 & 1403 & 76.64 & 64.39 \\
\hline 6 & 0 & 11,643 & 2,225 & 968 & 75.08 & 63.39 \\
\hline 7 & 0 & 11,448 & 13,504 & 1286 & 79.59 & 51.35 \\
\hline 8 & 18,000 & 11,353 & 30,300 & 1335 & 79.13 & 74.67 \\
\hline 9 & 18,000 & 11,346 & 36,837 & 1535 & 82.07 & 60.26 \\
\hline 10 & 16,000 & 11,504 & 33,594 & 1402 & 69.07 & 60.17 \\
\hline 11 & 36,000 & 11,348 & 34,556 & 1440 & 64.18 & 51.66 \\
\hline 12 & 15,000 & 11,264 & 30,186 & 1362 & 60.08 & 49.88 \\
\hline 13 & 40,000 & 11,238 & 35,567 & 1482 & 71.66 & 63.63 \\
\hline 14 & 36,000 & 11,430 & 33,311 & 1464 & 81.69 & 71.19 \\
\hline 15 & 0 & 11,649 & 15,583 & 729 & 78.63 & 65.93 \\
\hline 16 & 20,000 & 11,396 & 27,232 & 1295 & 68.44 & 56.75 \\
\hline 17 & 30,000 & 11,358 & 34,518 & 1443 & 79.71 & 65.69 \\
\hline 18 & 30,000 & 11,503 & 31,031 & 1320 & 79.37 & 68.64 \\
\hline 19 & 36,000 & 11,507 & 30,526 & 1296 & 77.83 & 64.01 \\
\hline 20 & 60,000 & 11,535 & 34,140 & 1423 & 77.71 & 64.91 \\
\hline 21 & 40,000 & 11,929 & 36,279 & 1512 & 79.04 & 75.90 \\
\hline 22 & 45,000 & 11,998 & 33,695 & 1446 & 77.92 & 58.08 \\
\hline 23 & 45,000 & 11,949 & 34,198 & 1439 & 74.56 & 54.90 \\
\hline 24 & 34,000 & 12,005 & 27,720 & 1171 & 74.71 & 62.76 \\
\hline 25 & 36,000 & 11,973 & 35,291 & 1470 & 74.88 & 61.21 \\
\hline 26 & 55,000 & 11,969 & 33,345 & 1389 & 73.08 & 61.42 \\
\hline 27 & 40,000 & 11,966 & 33,964 & 1415 & 74.92 & 64.35 \\
\hline 28 & 0 & 12,009 & 31,798 & 1325 & 73.78 & 70.19 \\
\hline 29 & 0 & 11,892 & 34,580 & 1492 & 77.04 & 72.10 \\
\hline 30 & 0 & 11,970 & 32,497 & 1449 & 78.12 & 61.23 \\
\hline AVERAGE/SUM & 745000 & 11616 & 920829 & 1362 & 76.28 & 61.23 \\
\hline
\end{tabular}

\section{Discussion and Anal ysis of Resul ts}

Tables 3 provides mill discharge size fractions, indicated as $-150 \mu \mathrm{m}$ discharge percentage (i.e. percentage of size fraction passing through $150 \mu \mathrm{m}$ sizing screen) for company 1 and 2 brand steel balls. Respective dried weight of particle size fraction retained on $150 \mu \mathrm{m}$ was measured by the use of digital weighing scale after performing sizing test on a given total sample weight. The percentage passing through $150 \mu \mathrm{m}$ was deduced by subtracting the weight retained on the $150 \mu \mathrm{m}$ screen from the given total sample weight for the test. The resultant weight (i.e. the weight of sample passing through $150 \mu \mathrm{m}$ screen) is expressed as percentage of the total sample weight. This gave the corresponding percentage passing through the $150 \mu \mathrm{m}$ screen of the various sample days. Statistically, the standard deviations for company 1 steel ball parameters are 4.82, 0.07 and 0.09 corresponding to $-150 \mu \mathrm{m}$ discharge percentage, steel ball consumption rate and power draw respectively. Similarly, company 2 steel ball brand has $6.48,0.13$ and 0.89 as standard deviation for $-150 \mu \mathrm{m}$ discharge percentage, steel ball consumption rate and power draw respectively. These relatively low standard de viations for the respective variables justify the use of the resultant average values (mean figures) as representative figures for the trade-off assessment. 
Table 3. Company 1 and 2 Steel Ball effect on Ball Mill Discharge Size fractions

\begin{tabular}{|c|c|c|c|c|c|c|}
\hline \multicolumn{2}{|c|}{ Company 1 Steel Ball } & \multicolumn{5}{|c|}{ Company 2 Steel Ball } \\
\hline No. Day & $\begin{array}{ll}-150 & \text { um } \\
\text { Discharge, } \%\end{array}$ & $\begin{array}{c}\text { Steel ball } \\
\text { consumption, } \\
\text { Kg/t }\end{array}$ & $\begin{array}{c}\text { Power/ton, } \\
\text { Kwh/t }\end{array}$ & $\begin{array}{c}-150 \text { um } \\
\text { Discharge, \% }\end{array}$ & $\begin{array}{c}\text { Steel ball } \\
\text { consumption, } \\
\mathrm{Kg} / \mathrm{t}\end{array}$ & $\begin{array}{l}\text { Power/ton, } \\
\text { Kwh/t }\end{array}$ \\
\hline 1 & 37.74 & 1.20 & 0.481 & 29.33 & 0.58 & 0.336 \\
\hline 2 & 41.36 & 1.31 & 0.328 & 33.62 & 0.58 & 0.335 \\
\hline 3 & 31.15 & 1.22 & 0.320 & 35.54 & 0.58 & 0.320 \\
\hline 4 & 38.43 & 1.20 & 0.339 & 34.89 & 0.56 & 0.398 \\
\hline 5 & 45.57 & 1.28 & 0.353 & 35.61 & 0.58 & 0.365 \\
\hline 6 & 40.13 & 1.25 & 0.364 & 36.61 & 0.57 & 5.233 \\
\hline 7 & 38.24 & 1.23 & 0.337 & 48.65 & 0.53 & 0.848 \\
\hline 8 & 49.22 & 1.22 & 0.355 & 25.33 & 0.54 & 0.375 \\
\hline 9 & 39.51 & 1.21 & 0.330 & 39.74 & 0.53 & 0.308 \\
\hline 10 & 41.29 & 1.17 & 0.344 & 39.83 & 0.52 & 0.342 \\
\hline 11 & 44.41 & 1.15 & 0.343 & 48.34 & 0.58 & 0.328 \\
\hline 12 & 40.56 & 1.12 & 0.341 & 50.12 & 0.57 & 0.373 \\
\hline 13 & 34.69 & 1.12 & 0.829 & 36.37 & 0.62 & 0.316 \\
\hline 14 & 37.74 & 1.05 & 0.357 & 28.81 & 0.66 & 0.343 \\
\hline 15 & 49.42 & 1.09 & 0.345 & 34.07 & 0.64 & 0.748 \\
\hline 16 & 43.87 & 1.08 & 0.306 & 43.25 & 0.64 & 0.418 \\
\hline 17 & 44.89 & 1.12 & 0.327 & 34.31 & 0.66 & 0.329 \\
\hline 18 & 38.68 & 1.13 & 0.323 & 31.36 & 0.68 & 0.371 \\
\hline 19 & 38.33 & 1.13 & 0.322 & 35.99 & 0.70 & 0.377 \\
\hline 20 & 42.79 & 1.12 & 0.329 & 35.09 & 0.77 & 0.338 \\
\hline 21 & 32.74 & 1.11 & 0.356 & 24.1 & 0.79 & 0.329 \\
\hline 22 & 34.94 & 1.10 & 0.304 & 41.92 & 0.81 & 0.356 \\
\hline 23 & 31.42 & 1.09 & 0.327 & 45.1 & 0.84 & 0.349 \\
\hline 24 & 46.97 & 1.09 & 0.384 & 37.24 & 0.85 & 0.433 \\
\hline 25 & 44.20 & 1.08 & 0.327 & 38.79 & 0.86 & 0.339 \\
\hline 26 & 40.47 & 1.07 & 0.330 & 38.58 & 0.89 & 0.359 \\
\hline 27 & 39.45 & 1.09 & 0.377 & 35.65 & 0.91 & 0.352 \\
\hline 28 & 42.20 & 1.08 & 0.397 & 29.81 & 0.87 & 0.378 \\
\hline 29 & 43.90 & 1.09 & 0.368 & 27.9 & 0.84 & 0.344 \\
\hline 30 & 46.97 & 1.09 & 0.418 & 38.77 & 0.81 & 0.368 \\
\hline Unit Value & 49.42 & 1.31 & 0.353 & $\mathbf{5 0 . 1 2}$ & 0.91 & 0.378 \\
\hline $\begin{array}{l}\text { Standard } \\
\text { Deviation }\end{array}$ & 4.82 & 0.07 & 0.09 & 6.48 & 0.13 & 0.89 \\
\hline
\end{tabular}

Tables 4 portrays the input and output cost evaluation indices for the two steel brands. Additionally, the respective power draw and calculated unit consumption per tonnage of ore milled are shown with their associated cost values. Company 1 steel ball brand has consumption of $1.31 \mathrm{~kg} / \mathrm{t}$ and power usage of $0.353 \mathrm{Kwh} / \mathrm{t}$ with cost of US\$1.62 and US\$0.06 respectively. These gave a resultant production cost of US\$1.68 for producing $49.42 \%$ particle size fraction passing through $150 \mu \mathrm{m}$ screen. Similarly, Company 2 steel ball brand has consumption of $0.91 \mathrm{~kg} / \mathrm{t}$ and power usage of $0.378 \mathrm{Kwh} / \mathrm{t}$ with cost of US\$1.23 and US\$0.07 respectively giving a total production cost of US\$1.30 for producing $50.12 \%$ particle size fraction passing through $150 \mu \mathrm{m}$ screen.

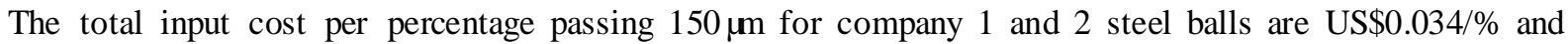
US $\$ 0.026 / \%$ respectively. Hence, percentage variance of $0.8 \%$ between total input cost per percentage passing $150 \mu \mathrm{m}(-150 \mu \mathrm{m}$ discharge) indicates the trade-off resulting from preferring one product (i.e. Company 1 or 2 steel balls) against the other. The relatively lower input cost per total input cost per percentage passing $150 \mu \mathrm{m}$ for company 2 as compared to that of company 1 steel balls point to a cost saving of $0.8 \%$ in favour of using company 2 steel balls. Conversely, using company 1 steel balls will generate a deficit of $0.8 \%$ as compared to company 2 steel balls.

Pragmatically, using company 1 steel ball brand for milling 36000 tons of ore per day at steel ball consumption rate of $1.31 \mathrm{~kg} / \mathrm{t}$ of ore implies a usage of 46.80 tons of steel balls per day. This translates into US\$57891.60 at US\$1237 per a ton of steel ball. Additionally, using $0.353 \mathrm{Kwh} / \mathrm{t}$ of power for milling 36000 tons of ore per day will generate a total power usage of $12708 \mathrm{Kwh}$ at a cost of US\$0.18 per Kwh. This implies total power cost of US\$2287.44 per day. Hence, the overall cost of producing $49.42 \%$ particle size fraction passing $150 \mu \mathrm{m}$ is US\$60051.60 per day. Conversely, applying trade-off of $0.80 \%$ (percentage variance) gave total amount to cost saving of US $\$ 480.00$ per day which will be equal to US\$175349.65 per year upon using company 2 steel ball brand instead of company 1 steel balls for milling. 
Table 4. Input and Output Cost evaluation indices for the two steel brands

\begin{tabular}{|c|c|c|c|c|c|}
\hline \multirow[b]{2}{*}{ Production Element } & \multirow[b]{2}{*}{ Cost element } & \multicolumn{2}{|c|}{ Company 1 Forged Steel Ball } & \multicolumn{2}{|c|}{ Company 2 Forged Steel Ball } \\
\hline & & Unit value & Cost, US\$ & Unit value & Cost, US\$ \\
\hline & Ste el Ball cons umption, $\mathrm{Kg} / \mathrm{t}$ & $\mathbf{1 . 3 1}$ & 1.62 & 0.91 & 1.23 \\
\hline$\Xi$ & Power per ton, Kwh/t & 0.353 & 0.060 & 0.378 & $\mathbf{0 . 0 7}$ \\
\hline$\overline{\underline{\Xi}}$ & Total Input Cost, US\$ & \multicolumn{2}{|c|}{1.68} & \multicolumn{2}{|r|}{1.30} \\
\hline & $-150 \mu \mathrm{m}$ discharge, $\%$ & 49.42 & 1.68 & 50.12 & 1.30 \\
\hline 言 & $\begin{array}{l}\text { Total Input cost per }-150 \mu \mathrm{m} \\
\text { discharge, US\$ } / \%\end{array}$ & \multicolumn{2}{|c|}{0.034} & \multicolumn{2}{|r|}{0.026} \\
\hline 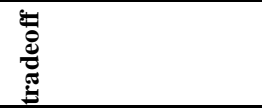 & $\begin{array}{l}\text { Total Input cost per }-150 \mu m \\
\text { discharge Variance, US\$/\% } \\
\text { Percentage Variance, } \%\end{array}$ & \multicolumn{4}{|c|}{0.008} \\
\hline
\end{tabular}

Analytically, decision matrix is one of the numerous format that can be used to assess the tradeoff of given items by evaluating the difference in total scores of respective weighted criteria for variables under consideration (Brady, 2011; Morán-Ordóñez, et.al, 2016; Yoe, 2002). Three input variable items used in assessing the trade-off for the two brands of steel brands are power cost, steel ball and $-150 \mu \mathrm{m}$ discharge percentage. Table 5 shows various trade-off obtained under the assumption of changes in cost of power usage with Company 2 steel ball total input cost per $-150 \mu \mathrm{m}$ discharge value as the reference point. Assessing the trade-off at assumed changes in power cost per kilowatt hour (Kwh) of US\$ 0 , US\$5, US\$10, US $\$ 15$, US\$20, US\$25 and US\$30 generate trade-off (Percentage variance) values of $0.850 \%, 0.650 \%, 0.451 \%, 0.251 \%, 0.052 \%,-0.148 \%$ and $-0.347 \%$ respectively. From Sharma (2011), figure 1 shows the linear trend with the trade-off in percentage variance as $\mathrm{Y}$ (dependent variable) and power cost per kilowatt hour as $\mathrm{X}$ (independent variable). This gave $\mathrm{y}=-0.0399 \mathrm{x}+$ 0.8496 as linear equation. Deduction from the given equation shows that, increasing power cost per kilowatt hour higher than US\$21.29, the trade-off will be negative. This implies that, at power cost per kilowatt hour higher than US\$21.29, it will be beneficial to buy or use company 1 brand of steel balls instead of buying company 2 steel balls. Similarly, all things other things being equal, from table 6 , the trade-off deductions under the assumption of changes in unit cost per ton of Company 1 steel ball brand at US\$500, US\$700, US\$900, US\$1100, US $\$ 1300$, US\$1500 and US $\$ 1700$ generate trade-off (Percentage variance) values of $0.011 \%, 0.006 \%$, $0.001 \%,-0.005 \%,-0.010,-0.015 \%$ and $-0.021 \%$ respectively. Figure 2 shows the linear trend with the trade-off in percentage variance as $\mathrm{Y}$ (dependent variable) and unit cost per ton of Company 1 steel ball brand as $\mathrm{X}$ (independent variable). This gave $\mathrm{y}=-0.0027 \mathrm{x}+2.4365$ as the linear equation. Deduction from the given equation shows that, at unit price above US\$902.41 per ton for company 1 steel ball brand, the trade-off will be negative. This implies that, at unit price above US\$902,41per ton for company 1 steel ball brand, it will be beneficial or profitable to buy or use company 2 brand of steel balls instead of buying company 1 steel balls. Subjecting the changes in price of power and the two steel ball brands to linear regression analysis show graphs with $\mathrm{r}^{2}=1$ which is a perfect reliable relation for forecasting or prediction.

However, from table 7 various trade-off obtained under the assumption of changes in unit cost per ton of Company 2 steel ball brand with the corresponding total input cost per $-150 \mu \mathrm{m}$ discharge value with cost per ton of Company 1 as the reference point. Assessing the trade-off at assumed changes in unit cost per ton of Company 2 steel ball brand of US $\$ 1300$, US $\$ 1400$, US $\$ 1500$, US $\$ 1600$, US $\$ 1700$, US $\$ 1800$ and US $\$ 1900$ generating trade-off (Percentage variance) values of $0.91146 \%, 0.72989 \%, 0.54833 \%, 0.3667 \%, 0.18520 \%, 0.00364 \%$ and $-0.17793 \%$ respectively. Figure 3 shows the linear trend with the equation $y=-0.0018 x+3.2718$ The $\mathrm{Y}$ (dependent variable) axis represents trade-off in percentage variance as and unit cost per ton of Company 2 steel ball brand is denoted as X (independent variable) axis (Sharma, 2011; Nau, 2014). Deduction from the given equation shows that, at unit price higher than US\$1817.67 per ton for company 2 steel ball brand, the trade -off will be negative. This implies that, at unit price higher than US $\$ 1817.67$ per ton for company 2 steel ball brand, it will be beneficial or profitable to buy or use company 1 brand of steel balls at a price US\$ 1237 per ton instead of buying company 2 steel balls. 
Table 5. Trade-off at variable prices for power input

\begin{tabular}{|c|c|c|c|c|c|c|c|c|c|}
\hline & & Peroids & 1 & 2 & 3 & 4 & 5 & 6 & 7 \\
\hline \multirow[t]{7}{*}{$\begin{array}{c}\text { Input } \\
\text { Element }\end{array}$} & \multicolumn{2}{|r|}{$\begin{array}{c}\text { Change Power cost per Kwh, } \\
\text { US\$ }\end{array}$} & $\mathbf{0}$ & 5 & 10 & 15 & 20 & 25 & 30 \\
\hline & \multirow{8}{*}{ 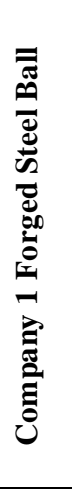 } & $\begin{array}{l}\text { Unit cost per ton of Ste el } \\
\text { Ball, US\$ }\end{array}$ & 1237 & 1237 & 1237 & 1237 & 1237 & 1237 & 1237 \\
\hline & & $\begin{array}{l}\text { Steel Ball consumption, } \\
\mathrm{Kg} / \mathrm{t}\end{array}$ & 1.31 & 1.31 & 1.31 & 1.31 & 1.31 & 1.31 & 1.31 \\
\hline & & $\begin{array}{l}\text { Steel Ball consumption } \\
\text { cost, US\$ }\end{array}$ & 1.62047 & 1.62047 & 1.62047 & 1.62047 & 1.62047 & 1.62047 & 1.62047 \\
\hline & & $\begin{array}{l}\text { Power usage per ton, } \\
\text { Kwh/t }\end{array}$ & 0.353 & 0.353 & 0.353 & 0.353 & 0.353 & 0.353 & 0.353 \\
\hline & & Power cost per ton, US\$ & $\mathbf{0}$ & 1.765 & 3.53 & 5.295 & 7.06 & 8.825 & 10.59 \\
\hline & & Total Input Cost, US\$ & 1.62047 & $\mathbf{3 . 3 8 5 4 7}$ & 5.15047 & 6.91547 & 8.68047 & 10.4455 & 12.2105 \\
\hline \multirow{2}{*}{$\begin{array}{l}\text { Output } \\
\text { Element }\end{array}$} & & $-150 \mu \mathrm{m}$ dis charge, $\%$ & 49.42 & 49.42 & 49.42 & 49.42 & 49.42 & 49.42 & 49.42 \\
\hline & & $\begin{array}{l}\text { Total Input cost per } \\
-150 \mu \mathrm{m} \\
\text { US\$ } \$ \%\end{array}$ & 0.03279 & 0.0685 & 0.10422 & 0.13993 & 0.17565 & 0.21136 & 0.24708 \\
\hline Input & \multirow{9}{*}{ 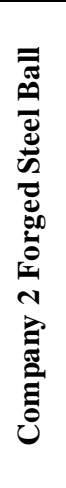 } & Unit cost per ton of Steel & 1338 & 1338 & 1338 & 1338 & 1338 & 1338 & 1338 \\
\hline \multirow[t]{6}{*}{ Element } & & Ball, US\$ & & & & & & & \\
\hline & & $\begin{array}{l}\text { Steel Ball consumption, } \\
\mathrm{Kg} / \mathrm{t}\end{array}$ & 0.91 & 0.91 & 0.91 & 0.91 & 0.91 & 0.91 & 0.91 \\
\hline & & $\begin{array}{l}\text { Steel Ball consumption } \\
\text { cost, US\$ }\end{array}$ & 1.21758 & 1.21758 & 1.21758 & 1.21758 & 1.21758 & 1.21758 & 1.21758 \\
\hline & & $\begin{array}{l}\text { Power usage per ton, } \\
\text { Kwh/t }\end{array}$ & 0.378 & 0.378 & 0.378 & 0.378 & 0.378 & 0.378 & 0.378 \\
\hline & & Power cost per ton, US\$ & $\mathbf{0}$ & 1.89 & 3.78 & 5.67 & 7.56 & 9.45 & 11.34 \\
\hline & & Total Input Cost, US\$ & 1.21758 & 3.10758 & 4.99758 & 6.88758 & 8.77758 & 10.6676 & 12.5576 \\
\hline \multirow{2}{*}{$\begin{array}{l}\text { Output } \\
\text { Element }\end{array}$} & & $-150 \mu \mathrm{m}$ dis charge, $\%$ & 50.12 & 50.12 & 50.12 & 50.12 & 50.12 & 50.12 & 50.12 \\
\hline & & $\begin{array}{l}\text { Total Input cost per } \\
-150 \mu \mathrm{m} \\
\text { US } \$ / \%\end{array}$ & 0.02429 & 0.062 & 0.09971 & 0.13742 & 0.17513 & 0.21284 & 0.25055 \\
\hline \multirow[t]{2}{*}{ Trade off } & \multirow{2}{*}{\multicolumn{2}{|c|}{$\begin{array}{c}\text { Total Input cost per }-150 \mu m \\
\text { discharge Variance (Trade } \\
\text { Off), US } \$ / \% \\
\text { Percentage Variance (Trade } \\
\text { Off), \% }\end{array}$}} & 0.0085 & 0.0065 & $\mathbf{0 . 0 0 4 5 1}$ & 0.00251 & 0.00052 & -0.00148 & -0.00347 \\
\hline & & & 0.850 & 0.650 & 0.451 & 0.251 & 0.052 & -0.148 & -0.347 \\
\hline
\end{tabular}

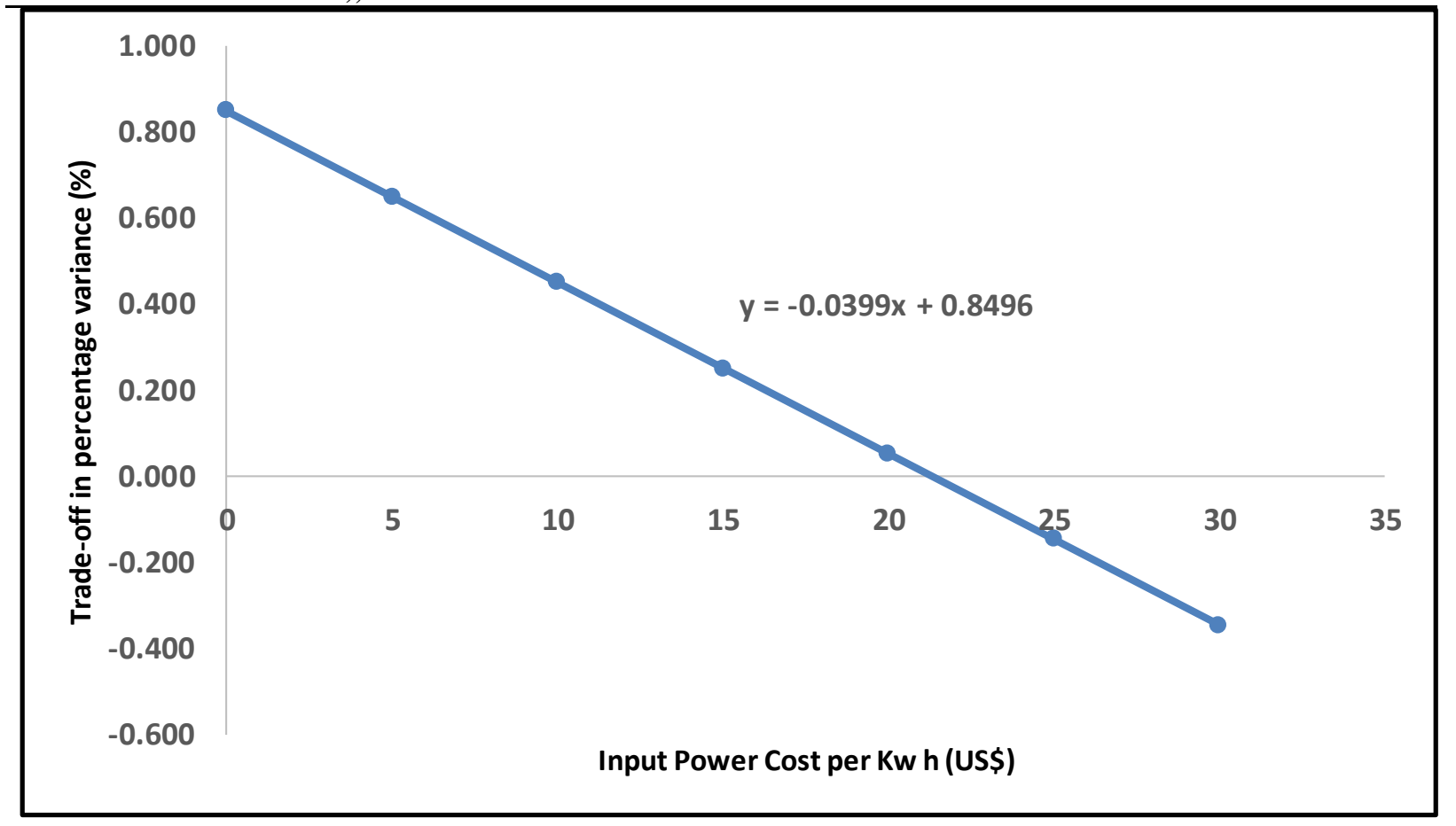

Figure 1. Trade-off relationship with variable power prices 
Table 6. Trade-off at variable prices for Company 1 steel ball brand input

\begin{tabular}{|c|c|c|c|c|c|c|c|c|c|}
\hline \multirow{3}{*}{$\begin{array}{c}\text { Input } \\
\text { Element }\end{array}$} & \multicolumn{2}{|r|}{ Peroids } & 1 & 2 & 3 & 4 & 5 & 6 & 7 \\
\hline & \multirow{9}{*}{ 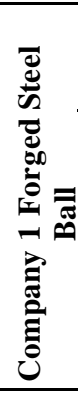 } & Power cost per Kwh, US\$ & 0.18 & 0.18 & $\mathbf{0 . 1 8}$ & 0.18 & $\mathbf{0 . 1 8}$ & $\mathbf{0 . 1 8}$ & 0.18 \\
\hline & & $\begin{array}{c}\text { Changes in Unit cost per ton of } \\
\text { Steel Ball, US\$ }\end{array}$ & 500 & 700 & 900 & 1100 & 1300 & 1500 & 1700 \\
\hline \multirow{7}{*}{$\begin{array}{l}\text { Output } \\
\text { Element }\end{array}$} & & Ste el Ball cons umption, $\mathrm{Kg} / \mathrm{t}$ & 1.31 & 1.31 & 1.31 & 1.31 & 1.31 & 1.31 & 1.31 \\
\hline & & Steel B all consumption cost, US\$ & 0.655 & 0.917 & 1.179 & 1.441 & 1.703 & 1.965 & 2.227 \\
\hline & & Power usage perton, $K w h / t$ & 0.353 & 0.353 & 0.353 & 0.353 & 0.353 & 0.353 & 0.353 \\
\hline & & Power cost per ton, US\$ & 0.064 & 0.064 & 0.064 & 0.064 & 0.064 & 0.064 & 0.064 \\
\hline & & Total Input Cost, US\$ & 0.719 & 0.981 & 1.243 & 1.505 & 1.767 & 2.029 & 2.291 \\
\hline & & $-150 \mu \mathrm{m}$ discharge, $\%$ & 49.42 & 49.42 & 49.42 & 49.42 & 49.42 & 49.42 & 49.42 \\
\hline & & $\begin{array}{l}\text { Total Input cost per }-150 \mu \mathrm{m} \\
\text { discharge, US } \$ \%\end{array}$ & 0.0145 & 0.0198 & 0.0251 & 0.0304 & 0.0357 & 0.0410 & 0.0463 \\
\hline \multirow[t]{6}{*}{$\begin{array}{c}\text { Input } \\
\text { Element }\end{array}$} & \multirow{5}{*}{ 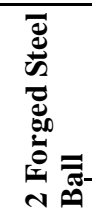 } & $\begin{array}{c}\text { Unit cost per ton of Steel Ball, } \\
\text { US\$ }\end{array}$ & 1338 & 1338 & 1338 & 1338 & 1338 & 1338 & 1338 \\
\hline & & Steel Ball cons umption, $\mathrm{Kg} / \mathrm{t}$ & 0.91 & 0.91 & 0.91 & 0.91 & 0.91 & 0.91 & 0.91 \\
\hline & & Steel Ball cons umption cost, US\$ & 1.2176 & 1.2176 & 1.2176 & 1.2176 & 1.2176 & 1.2176 & 1.2176 \\
\hline & & Power usage perton, Kwh/t & 0.378 & 0.378 & 0.378 & 0.378 & 0.378 & 0.378 & 0.378 \\
\hline & & Power cost per ton, US\$ & 0.068 & 0.068 & 0.068 & 0.068 & 0.068 & 0.068 & 0.068 \\
\hline & \multirow{3}{*}{ 己̇ } & Total Input Cost, US\$ & 1.286 & 1.286 & 1.286 & 1.286 & 1.286 & 1.286 & 1.286 \\
\hline \multirow{2}{*}{$\begin{array}{l}\text { Output } \\
\text { Element }\end{array}$} & & $-150 \mu \mathrm{m}$ discharge, $\%$ & 50.12 & 50.12 & 50.12 & 50.12 & 50.12 & 50.12 & 50.12 \\
\hline & & $\begin{array}{l}\text { Total Input cost per }-150 \mu \mathrm{m} \\
\text { discharge, US\$ } / \%\end{array}$ & 0.026 & 0.026 & 0.026 & 0.026 & 0.026 & 0.026 & 0.026 \\
\hline \multirow[t]{2}{*}{ Trade off } & \multirow{2}{*}{\multicolumn{2}{|c|}{ 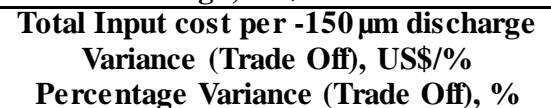 }} & 0.011 & 0.006 & 0.001 & -0.005 & -0.010 & -0.015 & -0.021 \\
\hline & & & 1.111 & 0.581 & 0.051 & -0.479 & -1.009 & -1.540 & -2.070 \\
\hline
\end{tabular}

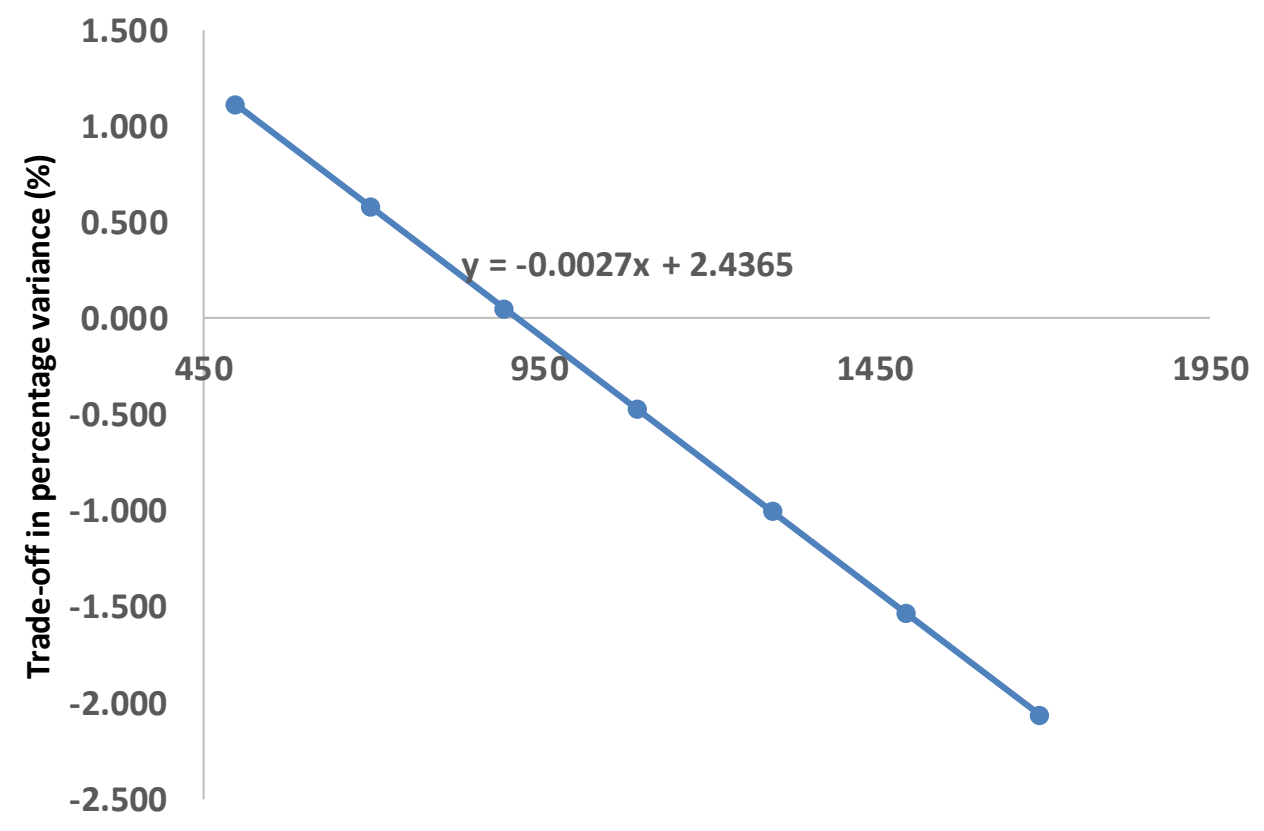

Company 1 Unit cost per ton of Steel Ball (US\$)

Figure 2. Trade-off relationship with variable Company 1 steel ball brand 
Table 7. Trade-off at variable prices for Company 2 steel ball brand input

\begin{tabular}{|c|c|c|c|c|c|c|c|c|c|}
\hline & & Peroids & 1 & 2 & 3 & 4 & 5 & 6 & 7 \\
\hline Input & \multicolumn{2}{|c|}{ Power cost per Kwh, US\$ } & 0.18 & 0.18 & 0.18 & 0.18 & 0.18 & 0.18 & 0.18 \\
\hline \multirow[t]{6}{*}{ Element } & \multirow{4}{*}{ 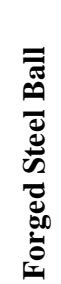 } & Changes in Unit cost per & 1237 & 1237 & 1237 & 1237 & 1237 & 1237 & 1237 \\
\hline & & $\begin{array}{l}\text { Steel Ball consumption, } \\
\text { Kg/t }\end{array}$ & 1.31 & 1.31 & 1.31 & 1.31 & 1.31 & 1.31 & 1.31 \\
\hline & & $\begin{array}{l}\text { Steel Ball consumption } \\
\text { cost, US\$ }\end{array}$ & 1.6205 & 1.6205 & 1.6205 & 1.6205 & 1.6205 & 1.6205 & 1.62047 \\
\hline & & $\begin{array}{l}\text { Power usage per ton, } \\
\text { Kwh/t }\end{array}$ & 0.353 & 0.353 & 0.353 & 0.353 & 0.353 & 0.353 & 0.353 \\
\hline & \multirow{3}{*}{ 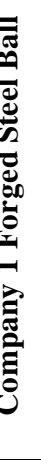 } & Power cost per ton, US\$ & 0.0635 & 0.0635 & 0.0635 & 0.0635 & 0.0635 & 0.0635 & 0.06354 \\
\hline & & Total Input Cost, US\$ & $\begin{array}{l}1.684 \\
49.42\end{array}$ & $\begin{array}{l}1.684 \\
49.42\end{array}$ & 1.684 & 1.684 & 1.684 & 1.684 & $\begin{array}{r}1.68401 \\
4942\end{array}$ \\
\hline Element & & $\begin{array}{l}\text { Total Input cost per } \\
-150 \mu \mathrm{m} \\
\text { US } \$ / \%\end{array}$ & 0.0341 & 0.0341 & 0.0341 & 0.0341 & 0.0341 & 0.0341 & 0.03408 \\
\hline \multirow[t]{5}{*}{$\begin{array}{c}\text { Input } \\
\text { Element }\end{array}$} & \multirow{5}{*}{ 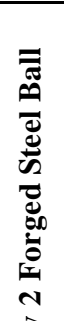 } & $\begin{array}{c}\text { Changes in Unit cost per } \\
\text { ton of Steel Ball, US\$ }\end{array}$ & 1300 & 1400 & 1500 & 1600 & 1700 & 1800 & 1900 \\
\hline & & $\begin{array}{l}\text { Steel Ball consumption, } \\
\mathrm{Kg} / \mathrm{t}\end{array}$ & 0.91 & 0.91 & 0.91 & 0.91 & 0.91 & 0.91 & 0.91 \\
\hline & & $\begin{array}{l}\text { Steel Ball consumption } \\
\text { cost, US\$ }\end{array}$ & 1.183 & 1.274 & 1.365 & 1.456 & 1.547 & 1.638 & 1.729 \\
\hline & & $\begin{array}{l}\text { Power usage per ton, } \\
\text { Kwh/t }\end{array}$ & 0.378 & 0.378 & 0.378 & 0.378 & 0.378 & 0.378 & 0.378 \\
\hline & & Power cost per ton, US\$ & 0.068 & 0.068 & 0.068 & 0.068 & 0.068 & 0.068 & 0.06804 \\
\hline \multirow{2}{*}{$\begin{array}{l}\text { Output } \\
\text { Element }\end{array}$} & \multirow{2}{*}{ 己ें } & $\begin{array}{l}\text { Total Input Cost, US\$ } \\
-150 \mu \mathrm{m} \text { discharge, } \%\end{array}$ & $\begin{array}{l}1.251 \\
50.12\end{array}$ & $\begin{array}{l}1.342 \\
50.12\end{array}$ & $\begin{array}{l}1.433 \\
50.12\end{array}$ & $\begin{array}{l}1.524 \\
50.12\end{array}$ & $\begin{array}{l}1.615 \\
50.12\end{array}$ & $\begin{array}{l}1.706 \\
50.12\end{array}$ & $\begin{array}{r}1.79704 \\
50.12\end{array}$ \\
\hline & & $\begin{array}{l}\text { Total Input cost per } \\
-150 \mu \mathrm{m} \\
\text { US } \$ / \%\end{array}$ & 0.02496 & 0.02678 & 0.02859 & 0.03041 & 0.03222 & 0.03404 & 0.03585 \\
\hline \multirow[t]{2}{*}{ Trade-off } & \multirow{2}{*}{\multicolumn{2}{|c|}{$\begin{array}{c}\text { Total Input cost per }-150 \mu m \\
\text { discharge Variance (Trade } \\
\text { Off), US } \$ / \% \\
\text { Percentage Variance (Trade } \\
\text { Off), \% }\end{array}$}} & 0.00911 & 0.00730 & 0.00548 & 0.00367 & 0.00185 & 0.00004 & -0.00178 \\
\hline & & & 0.91146 & 0.72989 & 0.54833 & 0.36677 & 0.18520 & 0.00364 & -0.17793 \\
\hline
\end{tabular}

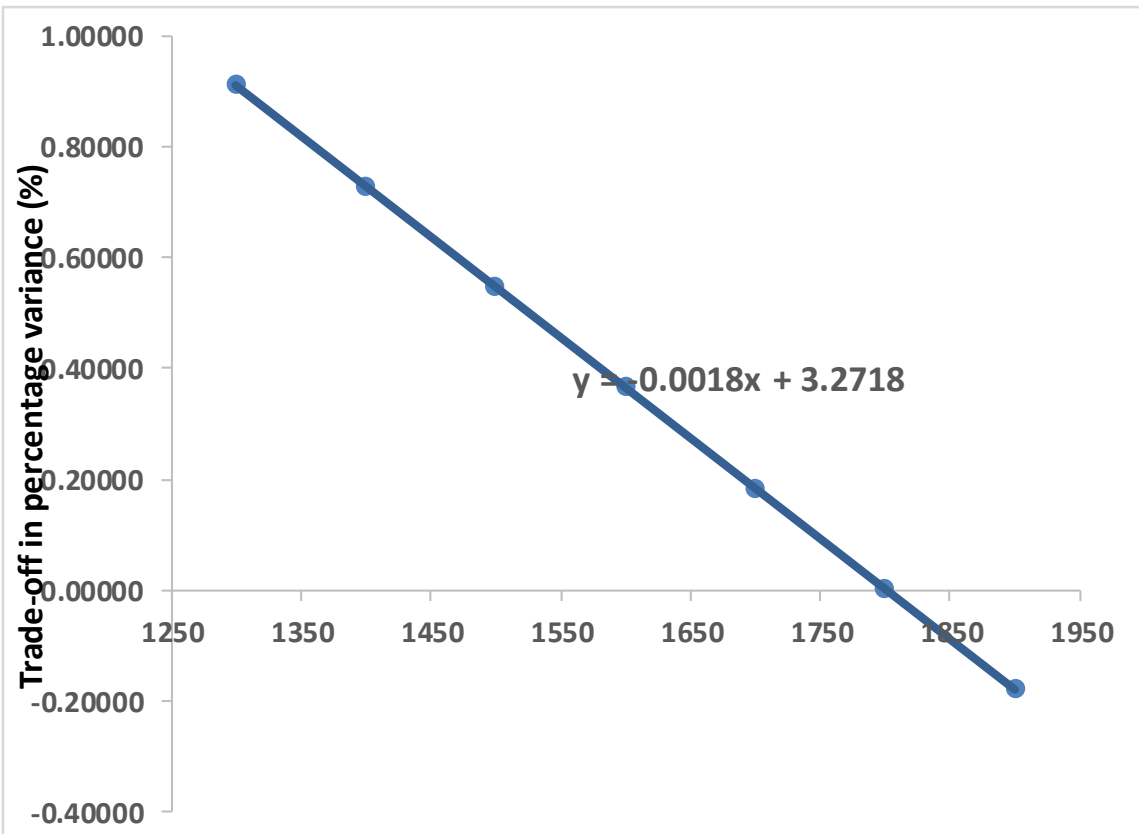

Company 2 Unit cost per ton of Steel Ball (US\$)

Figure 3. Trade-off relationship with variable Company 2 steel ball brand 


\section{Conclusion and Recommendation}

Trade-off has been decisive factor for detecting the focus of a given enterprise towards the achievement set goals of overcoming situations of compromising efficiency by trying to maximize profit under pretext of cheap price (Johnson, 2005; Hill, 2015). The quantitative analysis in this research was to test the two different brands of steel balls and their corresponding index numbers of the discharge product variables as a model for trade-off evaluation. The study illustrated the evaluations of power usage, steel ball consumption rate and resultant effects of the two different steel ball brands on the discharge product particle size fractions. From the data analysis, $49.42 \%$ particle size fraction passing $150 \mu \mathrm{m}$ by using company 1 steel ball brand as compared to $50.12 \%$ particle size fraction passing $150 \mu \mathrm{m}$ by using company 2 steel ball brand. Again, deduction from the total input cost per percentage passing $150 \mu \mathrm{m}$ for company 1 and 2 steel balls were US $\$ 0.034 / \%$ and US $\$ 0.026 / \%$ respectively. Hence, percentage variance of $0.8 \%$ between total input cost per percentage passing $150 \mu \mathrm{m}$ $(-150 \mu \mathrm{m}$ discharge) indicates the trade-off resulting from preferring one product (i.e. Company 1 or 2 steel balls) against the other. Application of the trade-off of $0.80 \%$ percentage variance, amount to cost saving of US\$480.00 per day which will be equal to US\$175349.65 per year upon using company 2 steel ball brand instead of company 1 steel balls for milling. Rationally, the research pointed out the shortfall of accepting input reagent (steel ball) of a production system on only the unit price or consumption rate variance between different items. The limitation of the research is the manual component of evaluating the size fractions of mill feed and discharge samples. Therefore, at the current conditions of unit price, it is recommendable and cost effective to use company 2 steel ball brand as compared to the company 1 steel ball brand. Again, by e valuating changes in price of power and the steel ball brands, company 2 steel ball should be preferred at unit cost of power lower than US\$21.29 and at unit prices lower than US\$1817.67per ton for company 2 steel ball brand. Also, further study using alternative method is recommended to enhance the prepositions made in this research work.

\section{References}

Bertsimas, D., Farias, V. F., \& Trichakis, N. (2012). On the Efficiency-Fairness Trade-off. Management science, 58(12), 2234. https://doi.org/10.1287/mnsc.1120.1549

Brady, S. (2011). How to: Completing a tradeoff analysis. https://www.prismdecision.com/how-to-complete-a-tradeoff-analysis/

Hill, A. (2015) Definition of Trade-Offs in Economics. http://study.com/academy/lesson/trade-offs-in-economics-definition-examples.html

Hornby, A. S. (2010). Oxford Advanced Learner's Dictionary of current English Eighth edition. Oxford University Press, U K. 313-1312.

Johnson, P. M. (2005). A Glossary of Political Economy Terms. http://www.auburn.edu/ johnspm/gloss/trade-off

Kettner, P. M., Moroney, R. M., \& Martin, L. L. (1990). Designing and managing programs: An effectiveness-based approach. 3rd edition Newbury Park, CA: Sage 5-8. https://www.amazon.com/Designing-Managing-Programs-Effectiveness-Based-Sourcebooks/dp/141295194 1

Morán-Ordóñez, A. et.al. (2016). Analysis of Trade-Offs Between Biodiversity, Carbon Farming and Agricultural Development in Northern Australia Reveals the Benefits of Strategic Planning. http://onlinelibrary.wiley.com/doi/10.1111/conl.12255/full

Nau, R. (2014). Notes on linear regression analysis 1. http://people.duke.edu/ rnau/notes_on_linear_regression_analysis--robert_nau.pdf

Saunders, M., Lewis P., \& Thornhill A. (2009). Research methods for business students fifth edition. Pearson Education, Limited, Edinbuegh Gate, Harlow, Essex CM20 2JEEngland. 465.

Sharma, H. (2011). Predictive Analytics \& Marketing - The next stage of Business Optimization, 25. http://www.optimizesmart.com/introducing-predictive-marketing-stage-business-optimization

Technical Report. (2016). Monthly Production Report. Metallurgy department of the Ghanaian Mine, Tarkwa Ghana. 1-6.

Weisbrod, B. A., \& Hansen, W. L. (1981). Benefits, Costs, and Finance of Public Higher Education. Markham. htt://en.wikipedia.org/wiki/cost benefit analysis.

Yoe, C. (2002). Trade-Off Analysis Planning and Procedures Guidebook 8-11. http://www.iwr.usace.army.mil/Portals/70/docs/iwrreports/02-R-2.pdf 
Young, J. (2013). Project trade-off analysis. http://www.skillpower.co.nz/2013/10/01/project-trade-off-analysis/

Zeckhauser, R. (1975). Procedures for valuing lives. Public Policy, 23(4), 419-464. http://www.ncbi.nlm.nih.gov/pubmed/1235897

\section{Copyrights}

Copyright for this article is retained by the author(s), with first publication rights granted to the journal.

This is an open-access article distributed under the terms and conditions of the Creative Commons Attribution license (http://creativecommons.org/licenses/by/4.0/). 